\title{
The correlation between white-matter microstructure and the complexity of spontaneous brain activity: A difussion tensor imaging-MEG study
}

\author{
Alberto Fernández ${ }^{\mathrm{a}, \mathrm{b}, *, 1}$, Marcos Ríos-Lago ${ }^{\mathrm{c}, \mathrm{d}, 1}{ }$, Daniel Abásolo ${ }^{\mathrm{e}}$, Roberto Hornero ${ }^{\mathrm{e}}$, Juan Álvarez-Linera ${ }^{\mathrm{d}, \mathrm{f}}$, \\ Nuria Paul ${ }^{\mathrm{a}}$, Fernando Maestú ${ }^{\mathrm{b}}$, Tomás Ortiz ${ }^{\mathrm{a}}$ \\ a Departamento de Psiquiatría y Psicología Médica, Universidad Complutense de Madrid, Spain \\ ${ }^{\mathrm{b}}$ Laboratorio de Neurociencia Cognitiva y Computacional, Centro de Tecnología Biomédica, Universidad Politécnica de Madrid y Universidad Complutense de Madrid, Spain \\ ' Departamento de Psicología Básica II, Universidad Nacional de Educación a Distancia (UNED), Madrid, Spain \\ ${ }^{\mathrm{d}}$ Unidad de Neuroimagen, Fundación CIEN-Fundación Reina Sofia.Madrid, Spain \\ e Grupo de Ingeniería Biomédica, Universidad de Valladolid, Spain \\ ${ }^{\mathrm{f}}$ Sección de Neurorradiología, Hospital Ruber Internacional, Madrid, Spain
}

Keywords:

Magentoencephalography

Difussion tensor imaging

Fractional anisotropy

Complexity

Brain oscillations

\begin{abstract}
A B S T R A C T
The advent of new signal processing methods, such as non-linear analysis techniques, represents a new perspective which adds further value to brain signals' analysis. Particularly, Lempel-Ziv's Complexity (LZC) has proven to be useful in exploring the complexity of the brain electromagnetic activity. However, an important problem is the lack of knowledge about the physiological determinants of these measures. Although a correlation between complexity and connectivity has been proposed, this hypothesis was never tested in vivo. Thus, the correlation between the microstructure of the anatomic connectivity and the functional complexity of the brain needs to be inspected. In this study we analyzed the correlation between LZC and fractional anisotropy (FA), a scalar quantity derived from diffusion tensors that is particularly useful as an estimate of the functional integrity of myelinated axonal fibers, in a group of sixteen healthy adults (all female, mean age $65.56 \pm 6.06$ years, intervals $58-82$ ). Our results showed a positive correlation between FA and LZC scores in regions including clusters in the splenium of the corpus callosum, cingulum, parahipocampal regions and the sagittal stratum. This study supports the notion of a positive correlation between the functional complexity of the brain and the microstructure of its anatomical connectivity. Our investigation proved that a combination of neuroanatomical and neurophysiological techniques may shed some light on the underlying physiological determinants of brain's oscillations.
\end{abstract}

\section{Introduction}

EEG and MEG signals derive from the collective and synchronous behavior of neural populations located in different brain structures (Cantero et al., 2009). As described by Segalowitz et al. (2010), EEG/ MEG signals are composed of the summation of multiple electrical oscillations at different frequencies. Therefore, the most common analysis procedure is spectral analysis, used to obtain the power of each frequency band. Although the underlying physiology of EEG/ MEG dynamics is not well understood (Robinson et al., 2001), at least some basic principles are acknowledged. For example, Pfurtscheller and Lopes da Silva (1999) posed three basic factors which determine the properties of EEG oscillations: 1 . the intrinsic membrane properties of the neurons and their synaptic processes, 2 . the strength of the interconnections between network elements, and 3 . the modulating influences from neurotransmitter systems.

Traditional spectral analysis provided researchers and clinicians with fundamental insights into brain function. However, the advent of new signal processing methods, particularly non-linear analysis techniques, represented a new perspective which added further value to brain signals' analysis (Babloyantz, 1983, Babloyantz, 1985). In his classic review on the neural dynamics of EEG, Lopes da Silva (1991) regarded EEG signals as generated by complex systems with non-linear dynamics. Since this basic concept was accepted by part of the scientific community, the number of non-linear estimates applied to EEG or MEG data is difficult to determine (see for a review Pereda et al., 2005; Stam, 2005). Among them, the estimates of signals' complexity are considered one of the most important fields of investigation.

The utilization of the term "complexity" is a problem per se (Heylighen, 1999). In fact, the definition of a system's complexity depends on so many factors that a common and broadly accepted description is difficult to reach. According to Tononi et al. (1998) definition, a system can be regarded as complex when there is a 
balance between regularity and randomness or between functional segregation and integration. Such theoretical approach crystallized in a measure, called neural complexity (CN) (Tononi et al., 1994), which has been used for the analysis of EEG/MEG data. Other definitions have been proposed. The correlation dimension (Babloyantz, 1985), one of the most frequently utilized complexity measures, seems to represent a non-linear estimate of the number of independent neuronal populations or oscillators which give rise to an EEG/MEG signal (Lutzenberger et al., 1995). Complexity definitions have also emerged from the field of information theory. For example, the algorithmic complexity (Kolmogorov, 1965) is defined as the length of the shortest computer program that generates a particular bit string. Similar to this, Lempel-Ziv's Complexity (LZC) is a non-linear measure for finite sequences related to the number of distinct substrings and the rate of their occurrence along the sequence, with larger values (in a $0-1$ scale) corresponding to more complexity in the data (Lempel and Ziv, 1976). Aboy et al. (2006) investigated the factors which affect LZC's values, and concluded that LZC represents an estimate of the number of different frequency components that actually compose the brain signals.

Whatever the complexity estimate utilized, and consequently whatever the definition of complexity adopted, an additional problem of these measures is the lack of knowledge about their physiological determinants. Since complexity estimates represent just an alternative procedure to analyze brain oscillations, it might be hypothesized that some of the basic physiological factors which determine the properties of those oscillations (i.e. membrane properties of the neurons, strength of the connections and modulating influences from neurotransmitter systems) should also determine complexity measures. To the best of our knowledge, only the correlation between connectivity and complexity has been investigated by means of computer simulations. Following up their interpretation of complexity as an equilibrium between functional segregation and integration, Tononi and Sporns' group (Sporns et al., 2000; Tononi et al., 1994) performed a simulation study in order to investigate the anatomical basis of functional integration and, more precisely, to elucidate which kind of anatomical substrate would yield a more complex functional outcome. According to their results using $\mathrm{CN}$, a network inspired in the visual cortex of the macaque monkey, thus ensuring the integrity of network's connectivity, yielded the highest complexity values. In their own words “...a necessary although not sufficient condition for complexity is high integration. In neuroanatomical terms, this means that a complex neural system must be highly interconnected" (Tononi et al., 1994). Recently, Barnett et al. (2009) confirmed these results.

However, some contradictory findings were also found. Karl Friston (1996) performed a simulation study trying to replicate the network characteristics of a "schizophrenic brain". A previous study showed higher complexity values in the EEG of patients with schizophrenia (Koukkou et al., 1993), and Friston tested the hypothesis that these higher complexity values were associated with a disconnection syndrome. According to his results, the more disconnected a network the higher correlation dimension values. These apparently contradictory findings may be explained not only by the different complexity estimate applied (a linear estimate such as CN vs. a non-linear estimate such as correlation dimension), but mainly by the lack of knowledge on the correlation between the "actual" integrity of the anatomic connectivity and the functional complexity of the brain.

Nowadays that correlation can be investigated by means of a combination of techniques, such as diffusion tensor imaging (DTI) and MEG. DTI is a MRI technique which makes possible in vivo exploration of microstructural features of white matter (WM) with quantitative methods (Basser and Pierpaoli, 1996; Beaulieu, 2002; Le Bihan and van Zijl, 2002). It measures both the rate and directionality of the displacement distribution of water molecules across tissue components on a voxel-by-voxel basis. The most commonly-used DTI-derived measure of WM microstructure is fractional anisotropy (FA), a scalar quantity derived from diffusion tensors that reflects the degree to which diffusion of water molecules is constrained in space due to local tissue properties, including fiber diameter and density, degree of myelination, and macrostructural features such as intravoxel fiber-tract coherence (Basser and Pierpaoli, 1996). The FA measure is particularly useful as an estimate of the microstructure and specific organization of myelinated axonal fibers. This measure ranges from zero, representing diffusion that is equal in all directions, to 1.0, representing complete directional dependence. FA tends to be higher in WM compared with gray matter (GM) due to the presence of myelinated fiber bundles, and it is greater for WM structures that contain the largest numbers of fibers running in parallel, such as the corpus callosum (Shimony et al., 1999).

Thus, we have performed an exploratory study where FA (derived from DTI) and LZC (derived from MEG) scores have been correlated in a group of healthy individuals. Following Tononi and Sporns' group interpretations (see above), we hypothesized that a positive correlation must exist between FA and LZC scores.

\section{Materials and methods}

\section{Participants}

Sixteen healthy adults (all female) were recruited from the community (mean age 65.56 \pm 6.06 years, intervals 58-82). Exclusion criteria consisted of current major Axis I psychiatric disease or history of major medical conditions (cancer, diabetes, or diagnosed neurological condition), as well as any previous diagnosis of substance dependence (excluding nicotine), or an undiagnosed pattern of behavior demonstrating longstanding maladaptive use of alcohol or other drugs. The initial sample was finally reduced to 14 subjects due to technical problems during the MRI analysis (see below).

All subjects provided written informed consent consistent with the Declaration of Helsinki. Of note, all participants remained intellectually active and participated in the educational program "University for seniors", at Complutense University.

\section{MEG data collection}

MEGs were acquired with a 148-channel whole-head magnetometer (MAGNES $2500 \mathrm{WH} \circledast$, 4D Neuroimaging, San Diego, CA) placed in a magnetically shielded room at "Centro de Magnetoencefalografía Dr. Pérez-Modrego" (Madrid, Spain). Subjects were in an awake but resting state with their eyes closed and under vigilance control during the recording. They were asked to avoid making eye movements. For each subject, five minutes of MEG signal were acquired at a sampling frequency of $678.17 \mathrm{~Hz}$ using a hardware band-pass filter of $0.1-200 \mathrm{~Hz}$. Afterwards these recordings were down-sampled by a factor of 4 $(169.549 \mathrm{~Hz}$ ). Artifact-free epochs of $20 \mathrm{~s}$ (see below) were selected offline. Finally, these epochs were filtered between 1.5 and $40 \mathrm{~Hz}$ and copied to a computer as ASCII files for further complexity analysis.

\section{LZC calculation}

LZC analysis is based on a coarse-graining of measurements. Therefore, the MEG signal must be previously transformed into a finite symbol string. In this study we used the simplest possible way: a binary sequence conversion (zeros and ones). By comparison with a threshold $T d$, the original signal samples are converted into a $0-1$ sequence $P=s(1), s(2), \ldots, s(n)$, with $s(i)$ defined by (Zhang et al., 2001):

$s(i)= \begin{cases}0 & \text { if } x(i)<T_{d} \\ 1 & \text { if } x(i) \geq T_{d}\end{cases}$

The threshold $T_{d}$ is estimated as the median value of the signals amplitude in each channel. We selected the median value because it is 
more robust to outliers (Nagarajan, 2002; Zhang et al., 2001). The string $P$ is then scanned from left to right and a complexity counter $c$ $(n)$ is increased by one unit every time a new subsequence of consecutive characters is encountered in the scanning process. The detailed algorithm for the measure of the $L Z$ complexity is as follows (Zhang et al., 2001):

1. Let $S$ and $Q$ denote two subsequences of the original sequence $P$ and $S Q$ be the concatenation of $S$ and $Q$ while $S Q \pi$ is a string derived from $S Q$ after its last character is deleted ( $\pi$ means the operation to delete the last character).

2. Let $v(S Q \pi)$ denote the vocabulary of all different substrings of $S Q \pi$.

3. At the beginning, the complexity counter $c(n)=1, S=s(1), Q=S$ (2), $S Q=s(1), s(2)$ and $S Q \pi=s(1)$.

4. In general, suppose that $S=s(1), s(2), \ldots, s(r), Q=s(r+1)$ and, therefore, $S Q \pi=s(1), s(2), \ldots, s(r)$. If $Q \in v(S Q \pi)$, then $Q$ is a subsequence of $S Q \pi$, not a new sequence.

5. $S$ does not change and renew $Q$ to be $s(r+1), s(r+2)$, then judge if $Q$ belongs to $v(S Q \pi)$ or not.

6. The steps 4 and 5 are repeated until $Q$ does not belong to $v(S Q \pi)$. Now $Q=s(r+1), s(r+2), \ldots, s(r+i)$ is not a subsequence of $S Q \pi=s(1), s(2), \ldots, s(r+i-1)$, so increase the counter by one.

7. Thereafter, $S$ and $Q$ are combined and renewed to be $s(1), s(2), \ldots, s$ $(r+i)$, and $s(r+i+1)$, respectively.

8. Repeat the previous steps until $Q$ is the last character. At this time, the number of different substrings is $c(n)$, the measure of complexity.

In order to obtain a complexity measure which is independent of the sequence length, $c(n)$ should be normalized. If the length of the sequence is $n$ and the number of different symbols is $\alpha$, it has been proved (Lempel and Ziv, 1976) that the upper bound of $c(n)$ is given by:

$c(n)<\frac{n}{\left(1-\varepsilon_{n}\right) \log _{\alpha}(n)}$

where $\varepsilon_{n}$ is a small quantity and $\varepsilon_{n} \rightarrow 0(n \rightarrow \infty)$. In general, $n / \log _{\alpha}(n)$ is the upper limit of $c(n)$, where the base of the logarithm is $\alpha$, i.e.,

$\lim _{n \rightarrow \infty} c(n)=b(n) \equiv \frac{n}{\log _{\alpha}(n)}$

For a binary conversion $\alpha=2$, and $b(n)$ is given by

$b(n) \equiv \frac{n}{\log _{2}(n)}$

and $c(n)$ can be normalized via $b(n)$ :

$C(n)=\frac{c(n)}{b(n)}$

$C(n)$ is usually a value between zero and one. The normalized LZC reflects the arising rate of new patterns along with the sequence (Zhang et al., 2001). A minimum data length must be considered to ensure that LZC reveals real data features (Yan and Gao, 2004). Since a previous work showed that the LZC values become stable for MEGs longer than 3000 samples (Gómez et al., 2006), an epoch length of 3392 data points $(20 \mathrm{~s})$ was used in the current study.

\section{MEG data reduction and analysis}

A LZC-normalized score was obtained for each channel and participant. Thus, 148 LZC scores per subject were submitted to statistical analyses. As in previous studies (Fernández et al., 2009, 2010) the initial 148 LZC scores were averaged into 5 regions: anterior, central, left lateral, right lateral, and posterior, which are

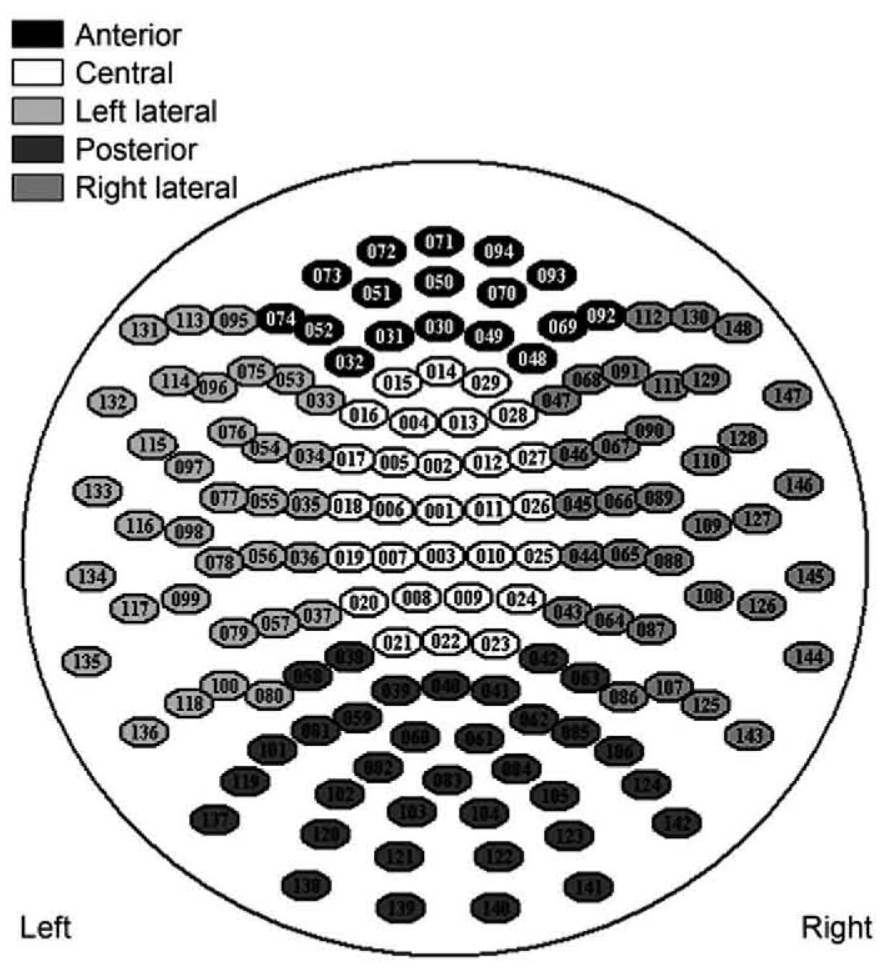

Fig. 1. Sensor-space representation of the five regions submitted to statistical analyses.

included as default sensor groups in the 4D-Neuroimaging source analysis software (see Fig. 1).

\section{DTI data acquisition}

Scanning was conducted on a 1.5T Signa Excite MR scanner (GE Healthcare, Waukesha, WI) using a customized DTI pulse sequence with an eight-channel head coil (GE Coils, Cleveland, OH). The sequence is based on a single-shot EPI pulse sequence with the capability of compensating eddy currents induced by the diffusion gradients via dynamically modifying the imaging gradient waveforms. Head motion was minimized with a vacuum-pack system molded to fit each subject. Nevertheless, the DTI exam could not be completed in two participants because of excessive motion. As a consequence DTILZC data analyses were performed on 14 subjects.

Whole-brain DTI was performed with a multislice single-shot spin echo echoplanar pulse sequence $(\mathrm{TE}=83 \mathrm{~ms}$, TR $=7000 \mathrm{~ms}$, flip angle of $90^{\circ}$ ) using 25 diffusion-encoding directions, isotropically distributed over the surface of a sphere with electrostatic repulsion, acquired at $\mathrm{b}=900 \mathrm{~s} / \mathrm{mm} 2,1$ acquisition with $\mathrm{b}=0 \mathrm{~s} / \mathrm{mm} 2,26$ slices of $5 \mathrm{~mm}$ thickness each with no gap between slices, a $256 \times 256$ matrix and a field of view (FOV) of $250 \mathrm{~mm}$. The slices were oriented in the intercommissural (AC-PC) plane.

An additional 3D high-resolution anatomical scan was also acquired: axial 3D inversion recovery fast spoiled gradient recalled echo (FSPGR) T1-weighted images ( $\mathrm{TE}=1.55 \mathrm{~ms}$, TR $=6.46 \mathrm{~ms}$, flip angle of $20^{\circ}, F O V=250 \mathrm{~mm}$, acquisition matrix $=256 \times 256$; slice thickness $=2 \mathrm{~mm}$ with $1 \mathrm{~mm}$ gap between slices.). All MR images were interpreted by attending neuroradiologists.

\section{DTI analysis}

The 25 diffusion directions, along with the $b=0$ image, were used to calculate FA as the primary indicator of WM microstructure. The images were reconstructed and FA was calculated using the program from Johns Hopkins, DTI Studio (Wakana et al., 2004). The 25 diffusion-weighted image sets were examined for image quality and 
head movement. Because noise can introduce bias in estimates of the eigenvalues and because noise decreases the signal-to-noise ratio, a background noise level was applied to all subjects prior to the calculation of pixelwise FA (background noise $=125$ ) (Kraus et al., 2007). The FA was then converted to ANALYZE format and read into Statistical Parametric Mapping software for analysis (SPM8, Wellcome Department of Imaging Neuroscience, London, UK, http://www. fil.ion.ucl.ac.uk/spm) implemented in Matlab (Mathworks, Natick, MA, USA). Then, the FA images were spatially normalized using the following normalization protocol. Firstly, the $b=0$ images of all participants were normalized to the standardized EPI template using linear transformations (Ashburner and Friston, 2000; Friston et al., 1995). After an initial affine transformation, an iterative non-linear normalization was applied using a lower threshold of $25 \mathrm{~mm}$ for the spatial periods of the discrete cosine basis functions by which brain warps are expanded in SPM (Ashburner and Friston, 2000). Secondly, the FA maps were then normalized using the parameters determined from the normalization of the $b=0$ image. From the resulting normalized data sets a study FA template was created by averaging, offering study-inherent contrast and distortions, according to the applied sequence parameters and additionally including the averaged anatomical characteristics of all participants. Then, all FA images were normalized again, but now using the study template that was created. Finally, all individually normalized FA data sets were smoothed by convolving them with an isotropic $6 \mathrm{~mm}$ FWHM (full width at half maximum) Gaussian kernel to improve the signal to noise ratio, to increase the validity of the statistical inference and to improve the normalization.

\section{Voxel-based analysis}

The linear correlation between FA and LZC scores was tested at each voxel with the multiple linear regression model implemented in SPM8. A voxelwise t-tests was performed to detect voxels where the slope of FA data against complexity measures as fitted by linear regression was significantly different from zero. In order to control a potential age influence, the variable "Age" was included as a covariate in the analysis. Inferences from statistical parametric maps were made at a significance level of $p<0.05$ FDR (False Discovery Rate) corrected for multiple comparisons across the entire brain (Genovese et al., 2002). This is an adaptive method to avoid false positives in such a way that they make up no more than $\alpha$ of the discoveries. The procedure calculates the uncorrected $\mathrm{p}$ value for each voxel and orders them so that the ordered $\mathrm{p}$ values are $P_{1} \leq P_{2} \leq \ldots \leq P_{N}$. To control the FDR at $\alpha$, it finds the largest value $k$ so that $P_{k}<\alpha k / N$. This procedure is less restrictive than Family Wise Error corrections (i.e. Bonferroni), but more sensitive, so it seems appropriate for an exploratory study because it allows power while maintains the control of type I error. For display purposes, thresholded t-maps were overlaid on the average of normalized FA images from the group for visualization (presented in neurological convention; $R=R$ ). For the labeling of the WM fiber tracts the DTI brain atlas by Mori et al. (2005) and Schmahmann and Pandya (2006) were used.

\section{Results}

\section{$L Z C$ regional analysis}

Differences of LZC variables across sensor regions were evaluated with a repeated-measures ANOVA with one factor ("Region" with 5 levels), and one covariate (Age). As in previous studies Age showed a significant effect on LZC variables $\left(F_{1,11}=5.721 ; p<0.05\right)$. However, Region did not exert a significant effect on $L Z C$ scores $\left(F_{4,44}=2.133\right.$; $\mathrm{p}=0.125$ ). Although LZC scores within central sensor group were slightly higher when compared to the other regions (see Table 1) the
Table 1

Means and standard deviations (SD) of the five sensor groups submitted to LZC analysis.

\begin{tabular}{llllll}
\hline & Anterior & Central & Left_lateral & Right_lateral & Posterior \\
\hline Mean & 0.6883 & 0.7204 & 0.6748 & 0.6856 & 0.6902 \\
SD & 0.0445 & 0.0359 & 0.0390 & 0.0435 & 0.0444 \\
\hline
\end{tabular}

regional effect was not statistically significant. Finally, the interaction Region $x$ Age was not significant $\left(\mathrm{F}_{4,44}=1.169 ; \mathrm{p}=0.168\right)$.

Considering these results we further inspected the relationship among sensor regions. A strong linear relationship was observed among the LZC scores in the five regions. All Pearson's correlation coefficients were in a range of 0.758 to 0.969 , with probabilities between $p=0.001$ and $p=0.0001$. This strong linear relationship among sensor groups' LZC scores implies that an average of all LZC scores is a suitable summary for the complexity of each individual (a very similar approach was utilized in Fernández et al., 2006). Thus, seeking the most parsimonious approach to explain the data, we will utilize an average of LZC scores in the correlation analyses with FA data.

\section{Correlation between FA and LZC scores}

Regions of cerebral WM containing voxels where FA was positively correlated with $\mathrm{LZC}$ measures are shown in Table 2. In all regions FA scores significantly decreased as LZC scores decreased (and FA increased as LZC increased). After controlling the effects of age, these regions included clusters bilaterally in the splenium of the corpus callosum (CC), the left parahipocampal region (cingulum), and left sagital stratum (inferior fronto-occiptal fasciculus and inferior longitudinal fasciculus) (see Table 2 and Fig. 2). No significant negative correlations were found in any case.

\section{Discussion}

The primary goal of this study was to investigate a hypothetical relationship between the functional complexity of the brain and the microstructure of its anatomic connectivity, using a combination of techniques such as LZC derived from MEG and FA derived from DTI. Our results confirmed a significant positive correlation between complexity and anatomical connectivity as represented by WM microstructure. When this correlation was first proposed by Tononi et al. (1994), and investigated by means of computer simulations, authors clearly claimed that a full validation of the hypothesis should be obtained "directly from brains in vivo". We tested the hypothesis in vivo, and this fact might be considered the main achievement of the study. However, since correlation analyses cannot determine a causal relationship between two variables, we cannot confirm a causal relationship between functional complexity and the microstructure of

\section{Table 2}

Clusters of voxels showing significant correlations between FA and LZ, and coordinates of the voxels with peak significance levels. Hemisphere $(H)$ : L: left; R: right. Cluster size: number of voxels in cluster.

\begin{tabular}{|c|c|c|c|c|c|c|c|}
\hline \multirow[t]{2}{*}{ Tract/bundle } & \multirow[t]{2}{*}{$\mathrm{H}$} & \multicolumn{3}{|c|}{ MNI coordinates } & \multirow{2}{*}{$\begin{array}{l}\text { Cluster } \\
\text { size }\end{array}$} & \multirow[t]{2}{*}{$\mathrm{t}$} & \multirow[t]{2}{*}{ Z } \\
\hline & & $\mathrm{X}$ & $\mathrm{Y}$ & $\mathrm{Z}$ & & & \\
\hline \multirow[t]{4}{*}{ Splenium of corpus callosum } & $\mathrm{R}$ & 6 & -24 & 22 & 104 & 10.27 & 5.00 \\
\hline & $\mathrm{L}$ & -8 & -30 & 22 & & 10.05 & 4.96 \\
\hline & $\mathrm{R}$ & 6 & -32 & 14 & & 8.74 & 4.69 \\
\hline & $\mathrm{L}$ & -24 & -58 & 14 & 4 & 7.62 & 4.41 \\
\hline $\begin{array}{l}\text { Sagittal stratum (include inferior } \\
\text { longitidinal fasciculus and } \\
\text { inferior fronto-occipital } \\
\text { fasciculus) }\end{array}$ & $\mathrm{L}$ & -36 & -80 & 2 & 5 & 8.76 & 4.69 \\
\hline $\begin{array}{l}\text { Cingulum (Parahippocampal } \\
\text { region) }\end{array}$ & $\mathrm{L}$ & -24 & -18 & -22 & 2 & 7.62 & 4.41 \\
\hline
\end{tabular}




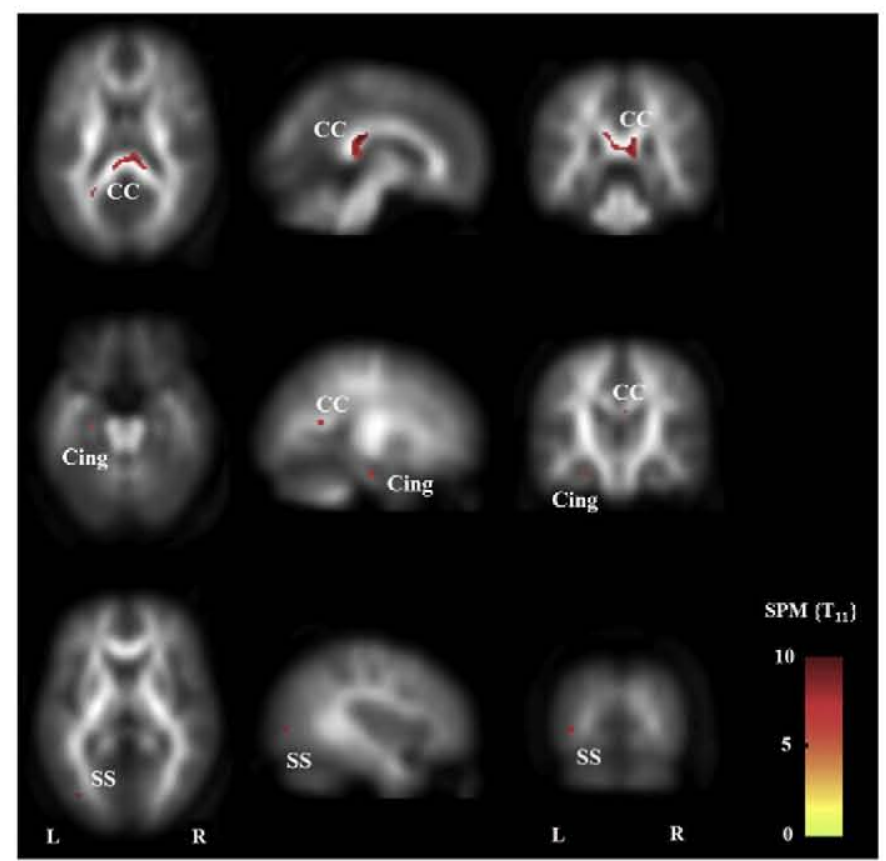

Fig. 2. White matter regions showing a positive correlation between FA and LZC (Sample size $=14$ ). Images thresholded at FDR $p<0.05$ and superimposed on the average normalized FA image of all participants. Neurological convention is followed (left side of the brain is shown on the left side of the figure). CC: Corpus Callosum (Splenium); Cing: Cingulum (Parahippocampal WM); SS: Sagittal Stratum.

the anatomical connectivity. We could rather affirm that an association exists between both processes.

In addition, our results indicate that a combination of MEG and DTI data seems to be adequate to analyze neurophysiological/anatomical correlations. Such combination has been recently utilized to investigate the visual and auditory evoked response (Roberts et al., 2009; Stufflebeam et al., 2008), epilepsy (Bhardwaj et al., 2010; Widjaja et al., 2010, 2009), traumatic brain injury (Huang et al., 2009), and language (Kamada et al., 2007); but never to examine a potential relationship between WM microstructure and an estimate of brain oscillations' frequency variability such as LZC. One of the most important aspects of neurophysiological/anatomical investigations is their potential role to untangle the physiological determinants of those oscillations. Despite this fact and the scientific importance of the problem (see Introduction), studies which specifically explore the "covariation" of neuroanatomy and neurophysiology are very scarce and recent. In their seminal study, Whitford et al. (2007) investigated how neuroanatomical changes (i.e. GM loss, synapic prunning, etc.) occurring in the transition between infancy and adolescence had a neurophysiological counterpart, represented by significant changes in the EEG spectrum. Their central hypothesis was, in essence, quite similar to the reasoning thread we posed in the introductory point of this study: since EEG power depends on a basic physiological process determined by the coherent synaptic activity of neural groups, a significant reduction in the number of synapses should produce, in turn, a significant reduction of the estimated EEG power. As Whitford and co-workers pointed out, such a "logical" covariation had never been directly investigated before. These authors found a significant positive correlation between GM volumes and their corresponding regional EEG powers, especially in the low-frequency bands. In other words, the curvilinear decrease of GM volume as a function of age was accompanied by a curvilinear decrease of absolute EEG power with age. Interestingly, WM volume was found to increase curvilinearly as a function of age, but this process was not found to correlate with any of the spectral measures. Whitford and co-workers' results not only confirmed their previous hypothesis but also opened a window to the perspective of two physiologically independent processes (those associated with GM and those associated with WM) which are undoubtedly regulating the properties of EEG/MEG signals. In their excellent review of the literature, Segalowitz et al. (2010) also highlighted the importance of this "logical" association between agerelated neuronatomical changes and their associated neurophysiological changes, and addressed the need of further direct investigations.

Our results suggest that while conventional spectral measures, such as power spectrum, seem to be associated with GM development (Whitford et al., 2007); non-linear estimates of complexity, such as LZC scores, seem to be associated with the process of WM development in the brain. This notion is supported by the parallel tendencies of WM and complexity evolution reported in some studies. For example, Anokhin et al. (1996) and Meyer-Lindenberg (1996) demonstrated that while EEG power shows a progressive decrease as a function of age, correlation dimension values increase linearly within the age interval of 7 to 60 years. Similarly, while neuroanatomical studies on GM development show a progressive reduction of its volume which starts in childhood (Giedd et al., 1999; Sowell et al., 1999; Steen et al., 1997), WM investigations tend to exhibit an opposite tendency (Ben Bashat et al., 2005; Gao et al., 2009; Hasan et al., 2009, 2007). These parallel trajectories of WM and complexity values might appear as apparently broken during late adulthood, since some studies (see for example McLaughlin et al., 2007) demonstrated a FA peak in young adulthood followed by a decline. On the contrary, Anokhin et al. (1996) and Meyer-Lindenberg (1996) found a continuous increase of complexity with age evaluating the resting EEGs of 5 age groups from 7 to 60 years. In a recent study (Fernández et al., 2010), we suggested that such uninterrupted complexity increase might be explained by sample's characteristics. The upper age limit in our study was increased to include subjects in their late eighties and a significant, linear decrease of complexity scores was observed from adulthood to senescence (Fernández et al., 2010).

At this point we must address the potential clinical relevance of the finding. LZC has been successfully used to analyze EEG and MEG signals in patients with Alzheimer's disease (Abásolo et al., 2006; Fernández et al., 2010; Gómez et al., 2006), attention deficithyperactivity disorder (Fernández et al., 2009), depression and schizophrenia ( $\mathrm{Li}$ et al., 2008) as well as to measure the depth of anesthesia (Ferenets et al., 2007; Zhang et al., 2001), or to study epileptic seizures (Radhakrishnan and Gangadhar, 1998). Overall, LZC scores decreased in those pathologies where some type of connectivity dysfunction was expected, while such dysfunction could be originated by some type of disconnection syndrome or by an abnormal maturation of WM tracts. For instance, LZC scores were significantly lower in Alzheimer's disease (Abásolo et al., 2006; Gómez et al., 2006) and this fact was accompanied by an exaggerated reduction of patients' complexity scores with age, as compared to controls (Fernández et al., 2010). The reduction of LZC scores was attributed to the well-known disconnection syndrome in Alzheimer's disease and the consequent connectivity impairment observed in these patients (Bozzali et al., 2002; Hirono et al., 2000; Stam et al., 2007). Similarly, LZC was found to be abnormally reduced in a group of attention deficit/hyperactivity disorder patients as compared to healthy children (Fernández et al., 2009). Furthermore, the agerelated trajectory of LZC was divergent in both groups. LZC tended to increase in healthy controls, while patients exhibited a nonsignificant tendency to reduced complexity scores as a function of age. Such divergent maturational trajectory was interpreted as a new illustration of neurodevelopmental deficits in attention deficit/ hyperactivity disorder, which basically affect the process of WM maturation (Shaw et al., 2007). Alzheimer's disease and attention deficit/hyperactivity disorder are two examples of the potential clinical role of complexity estimates. Some other cases of similar 
relevance, where a disconnection syndrome or an abnormal WM maturation have been hypothesized, are schizophrenia (see Ho et al., 2003; Jones et al., 2006) or autism spectrum disorders (Cheng et al., 2010). Interestingly, all the mentioned disorders are characterized by severe cognitive and behavioral manifestations.

The main goal of this study is not an exhaustive anatomical discussion on the observed correlations between FA and LZC. Nevertheless, it worths some remarks. Correlations have been found in regions including commissural (Corpus Callosum), projection (Sagittal stratum), and association fibers (cingulum/parahippocampal WM), predominantly in posterior regions of the left hemisphere. The splenium of the CC, where FA is greater (Chepuri et al., 2002), is traversed by three functionally specialized groups of myelinated fibers that bidirectionally connect parietal, temporal and occipital lobes with a roughly ordered arrangement of axons according to their origin (Aboitiz and Montiel, 2003; Huang et al., 2005; Dougherty et al., 2007; Zarei et al., 2006; Hasan et al., 2009). Fibers from the occipital lobe, from the lateral and caudal portions of the parahippocampal gyrus and from the caudal temporal lobe travel through the splenium, where our "target" correlation areas seem to be located. Whether our results are showing correlations between complexity and fibers from any of these parietal, occipital or temporal areas needs to be elucidated. The CC (being the primary interhemispheric pathway) is basic for all sensory and high-level cognitive integration, and its posterior region could give anatomical support to the clusters of dense connectivity reported by Hagmann et al. (2008). These authors found evidence for the existence of a structural core composed of posterior medial and parietal cortical regions that are densely interconnected. The high degree of interhemispheric coupling within the core suggests that it acts as a single integrated system form which processes in both cortical hemispheres are coordinated. The correlation between complexity measures and FA in the splenium of the CC could reflect that this structure would be responsible for integrating information across functionally segregated brain regions. Its anatomical correspondence with some elements of the human default network suggests that the core may be an important structural basis for shaping large-scale brain dynamics (Fox and Raichle, 2007; Hagmann et al., 2008; Raichle et al., 2001).

With regard to fibers connecting the parahippocampal gyri and the hippocampal formation (often associated with memory and emotion), it has been shown to send relatively dense heterotopic connections to the posterior part of the superior temporal gyrus, the planum temporale, and the supramarginal and the angular gyri; as well as weak connections to the posterior part of the inferior frontal gyrus (Di Virgilio and Clarke, 1997; Markowithsch, 1995). As with the CC, it is necessary to study in detail which of these associations is more closely connected with the complexity measures. However, Bonifazi et al. (2009) demonstrated the existence of neurons within the hippocampus, with a widespread axonal arborisation and long-range connections that link a large number of cells, working as functional hubs (superconnected nodes). This kind of architecture and network connectivity might underlie the complexity measures reported in our study.

Finally, the Sagittal stratum is a major corticosubcortical bundle that conveys fibers from the parietal, occipital, cingulate, and temporal regions to subcortical destinations in the thalamus, the nuclei of the basis pontis, and other brainstem structures. It also conveys afferents from the thalamus to the cortex. It may therefore be considered as equivalent to the internal capsule at the posterior part of the hemispheres (Schmahmann and Pandya, 2006). According to the left lateralization of the results, they converge with those provided by Hagmann et al. (2008) who show that the structural core was located within posterior medial cortex, and extended laterally into parietal and temporal cortices, especially in the left hemisphere.

From our point of view, these widespread connections support the role of complexity estimates in the investigation of those disorders with cognitive and behavioral manifestations. Any compromise of these connections would highly influence the entire network dynamics.

This study is no exception in having limitations. The diffusion tensor represents an average of the tissue sampled, and it is likely that with our voxel size and shape some partial volume artifact is present. Furthermore, a voxel may contain small tracts with differing directions, resulting in a misleading average impression of tract direction. In such areas of incoherent fiber trajectories, anisotropy decreases, and FA could be understimated. According to Oouchi et al. (2007), when anisotropic voxels are used, the FA measured in areas without crossing fibers is not affected. However, it is understimated in other areas of the brain with crossing fibers. The obtention of small clusters outside the corpus callosum, once the correction for multiple comparisons was applied, may be due to this FA error estimation. It is possible that this error remains constant across subjects, so a correlation analysis should be less affected, but this would only be true if the normalization process was perfect.

The normalization and spatial filtering processes have certain limitations when conducting a voxel-based analysis (Camara et al., 2007; Jones et al., 2005). The normalization and the small Gaussian smoothing kernell selected may also have contributed to the identified correlations between FA and complexity measures. In order to minimize intersubject anatomical variations as much as possible we used an optimized normalization protocol including the creation of a suitable internal-study brain template (Good et al., 2001) to facilitate the characterization of microstructural correlations at a voxel level. However, great care must be exercised both in obtaining and in interpreting DTI data. Anatomically defined regions-of-interest may be used to corroborate results from voxel-based analysis, allowing white matter to be tested without the influence of the normalization process (Salat et al., 2005; Camara et al., 2007). Despite these, it is clear that DTI is still a highly sensitive method for the evaluation of the fiber tracts microstructure. Therefore, as long as researchers are aware of possible artifacts arising from these methodological constraints, voxel-based analysis is a useful approach for identifying a possible relation between white matter and MEG complexity measures across the whole brain.

\section{Conclusions}

The observed significant positive correlations between LZC and FA were obtained from a sample with a relatively small size, and this may limit the generalization of our results.

Nevertheless, this piece of work is an exploratory investigation which supports the notion of a positive correlation between the functional complexity of the brain and the microstructure of its anatomical connectivity. As we previously pointed out, it seems that WM and GM changes across the lifespan exert a clear but divergent influence on brain's oscillatory activity. GM changes seem to modify the spectral properties such as absolute power (Whitford et al., 2007), while WM changes seem to affect the non-linear properties of brain oscillations, here represented by complexity estimates. This conclusion not only confirms Tononi and Sporns' group hypothesis about complexity and connectivity but also upholds a broader point of view about the complexity of biological systems. In his fine review on the evolutionary concept of complexity, Heylighen (1999) described the evolution as a process where more complex organisms (or biological systems) evolve from more simple ones. Which is the critical characteristic of this complexity increase? More complex systems are typically composed of several "distinct" parts but, essentially, those parts must be closely connected at different levels to ensure the proper system's functioning. The clinical experience reveals that a dysfunctional connectivity in the brain (the most complex biological system) is associated with severe cognitive and behavioral impairments, and we are now beginning to understand that it is also associated with a reduced 
functional complexity. Our results might shed some light on the underlying physiological determinants of this process.

\section{Acknowledgments}

This study was partially funded by the Madr.IB (CAM $i+d+I$ project; S-SAL-0312-2006) and the Spanish Ministry of ScienceSEJ2006-07560, PSI2009-14415-C03-01, and PSI2009-14415-C03-03.

\section{References}

Abásolo, D., Hornero, R., Gómez, C., Garcia, M., Lopez, M., 2006. Analysis of EEG background activity in Alzheimer's disease patients with Lempel-Ziv complexity and central tendency measure. Med. Eng. Phys. 28 (4), 315-322.

Aboitiz, F., Montiel, J., 2003. One hundred million years of interhemispheric communication: the history of the corpus callosum. Braz. J. Med. Biol. Res. 36 (4), $409-420$.

Aboy, M., Hornero, R., Abásolo, D., Alvarez, D., 2006. Interpretation of the Lempel-Ziv complexity measure in the context of biomedical signal analysis. IEEE Trans. Biomed. Eng. 53 (11), 2282-2288.

Anokhin, A.P., Birbaumer, N., Lutzenberger, W., Nikolaev, A., Vogel, F., 1996. Age increases brain complexity. Electroencephalogr. Clin. Neurophysiol. 99 (1), 63-68.

Ashburner, J., Friston, K.]., 2000. Voxel-based morphometry-the methods. NeuroImage $11,805-821$.

Babloyantz, A., 1983. Estimation of correlation dimension from single and multichannel recordings: a critical review. In: Basar, E.B.T.H. (Ed.), Brain Dynamics. Springer, Berlin, pp. 122-131.

Babloyantz, A., 1985. Evidence of chaotic dynamics of brain activity during the sleep cycle. Phys. Lett. (A) 111, 152-156.

Barnett, L., Buckley, C.L., Bullock, S., 2009. Neural complexity and structural connectivity. Phys. Rev. E Stat. Nonlin. Soft. Matter. Phys. 79 (5 Pt 1), 051914.

Basser, P.J., Pierpaoli, C., 1996. Microstructural and physiological features of tissues elucidated by quantitative-diffusion-tensor MRI. J. Magn. Reson. B 111 (3), $209-219$.

Beaulieu, C., 2002. The basis of anisotropic water diffusion in the nervous system - a technical review. NMR Biomed. 15 (7-8), 435-455.

Ben Bashat, D., Ben Sira, L., Graif, M., Pianka, P., Hendler, T., Cohen, Y., Assaf, Y., 2005. Normal white matter development from infancy to adulthood: comparing diffusion tensor and high $\mathrm{b}$ value diffusion weighted MR images. J. Magn. Reson. Imaging 21 (5), 503-511.

Bhardwaj, R.D., Mahmoodabadi, S.Z., Otsubo, H., Snead III, O.C., Rutka, J.T., Widjaja, E., 2010. Diffusion tensor tractography detection of functional pathway for the spread of epileptiform activity between temporal lobe and Rolandic region. Childs Nerv. Syst. $26(2), 185-190$.

Bonifazi, P., Goldin, M., Picardo, M.A., Jorquera, I., Cattani, A., Bianconi, G., Represa, A. Ben-Ari, Y., Cossart, R., 2009. GABAergic hub neurons orchestrate synchrony in developing hippocampal networks. Science 326 (5958), 1419-1424.

Bozzali, M., Cercignani, M., Sormani, M.P., Comi, G., Filippi, M., 2002. Quantification of brain gray matter damage in different MS phenotypes by use of diffusion tensor MR imaging. AJNR Am. J. Neuroradiol. 23, 985-988.

Camara, E., Bodammer, N., Rodriguez-Fornells, A., Tempelmann, C., 2007. Age-related water diffusion changes in human brain: a voxel-based approach. Neurolmage 34 (4), 1588-1599.

Cantero, J.L., Atienza, M., Cruz-Vadell, A., Suarez-Gonzalez, A., Gil-Neciga, E., 2009. Increased synchronization and decreased neural complexity underlie thalamocortical oscillatory dynamics in mild cognitive impairment. Neurolmage 46 (4), 938-948.

Cheng, Y., Chou, K.H., Chen, I.Y., Fan, Y.T., Decety, J., Lin, C.P., 2010. Atypical development of white matter microstructure in adolescents with autism spectrum disorders. Neurolmage 50 (3), 873-882.

Chepuri, N.B., Yen, Y.F., Burdette, J.H., Li, H., Moody, D.M., Maldjian, J.A., 2002. Diffusion anisotropy in the corpus callosum. AJNR Am. J. Neuroradiol. 23 (5), 803-808.

Di Virgilio, G., Clarke, S., 1997. Direct interhemispheric visual input to human speech áreas. Hum. Brain Mapp. 5 (5), 347-354.

Dougherty, R.F., Ben-Shachar, M., Deutsch, G.K., Hernandez, A., Fox, G.R., Wandell, B.A., 2007. Temporal-callosal pathway diffusivity predicts phonological skills in children. Proc. Natl. Acad. Sci. U. S. A. 104 (20), 8556-8561.

Ferenets, R., Vanluchene, A., Lipping, T., Heyse, B., Struys, M.M., 2007. Behavior of entropy/complexity measures of the electroencephalogram during propofolinduced sedation: dose-dependent effects of remifentanil. Anesthesiology 106 (4), 696-706.

Fernández, A., Hornero, R., Mayo, A., Poza, J., Gil-Gregorio, P., Ortiz, T., 2006. MEG spectral profile in Alzheimet's disease and mild cognitive impaiment. Clin. Neurophysiol. 117 (2), 306-314.

Femández, A., Quintero, J., Homero, R., Zuluaga, P., Navas, M., Gómez, C., Escudero, J., Garcia-Campos, N., Biederman, J., Ortiz, T., 2009. Complexity analysis of spontaneous brain activity in attention-deficit/hyperactivity disorder: diagnostic implications. Biol. Psychiatry 65 (7), 571-577.

Fernández, A., Hornero, R., Gómez, C., Turrero, A., Gil-Gregorio, P., Matias-Santos, J., Ortiz, T., 2010. Complexity analysis of spontaneous brain activity in Alzheimer disease and mild cognitive impairment: an MEG study. Alzheimer Dis. Assoc. Disord. 24 (2), 182-189.
Fox, M.D., Raichle, M.E., 2007. Spontaneous fluctuations in brain activity observed with functional magnetic resonance imaging. Nat. Rev. Neurosci. 8, 700-711.

Friston, K.J., 1996. Theoretical neurobiology and schizophrenia. Br. Med. Bull. 52 (3), $644-655$.

Friston, K.J., Ashburner, J., Frith, C., Poline, J.B., Heather, J.D., Frackowiak, R.S.J., 1995. Spatial registration and normalization of images. Hum. Brain Mapp. 2, 165-189.

Gao, W., Lin, W., Chen, Y., Gerig, G., Smith, J.K., Jewells, V., Gilmore, J.H., 2009. Temporal and spatial development of axonal maturation and myelination of white matter in the developing brain. AJNR Am. J. Neuroradiol. 30 (2), 290-296.

Genovese, C.R., Lazar, N.A., Nichols, T., 2002. Thresholding of statistical maps in functional neuroimaging using the false discovery rate. Neurolmage $15,870-878$.

Giedd, J.N., Blumenthal, J., Jeffries, N.O., Castellanos, F.X., Liu, H., Zijdenbos, A., Paus, T., Evans, A.C., Rapoport, J.L., 1999. Brain development during childhood and adolescence: a longitudinal MRI study. Nat. Neurosci. 2 (10), 861-863.

Gómez, C., Hornero, R., Abásolo, D., Fernández, A., López, M., 2006. Complexity analysis of the magnetoencephalogram background activity in Alzheimer's disease patients. Med. Eng. Phys. 28 (9), 851-859.

Good, C.D., Johnsrude, I.S., Ashburner, J., Henson, R.N., Friston, K.J., Frackowiak, R.S., 2001. Avoxel-based morphometric study of ageing in 465 normal adult human brains. Neurolmage 14, 21-36.

Hagmann, P., Cammoun, L., Gigandet, X., Meuli, R., Honey, C.J., Wedeen, V.J., Sporns, 0. 2008. Mapping the structural core of human cerebral cortex. PLoS Biol. 6 (7), e159.

Hasan, K.M., Sankar, A., Halphen, C., Kramer, L.A., Brandt, M.E., Juranek, J., Cirino, P.T. Fletcher, J.M., Papanicolaou, A.C., Ewing-Cobbs, L., 2007. Development and organization of the human brain tissue compartments across the lifespan using diffusion tensor imaging. Neuroreport 18 (16), 1735-1739.

Hasan, K.M., Kamali, A., Iftikhar, A., Kramer, L.A., Papanicolaou, A.C., Fletcher, J.M., Ewing-Cobbs, L., 2009. Diffusion tensor tractography quantification of the human corpus callosum fiber pathways across the lifespan. Brain Res. 1249, 91-100.

Heylighen, F., 1999. The Growth of Structural and Functional Complexity during Evolution. Kluwer Academic, Dordrecht, pp. 17-44.

Hirono, N., Kitagali, H., Kazui, H., Hashimoto, M., Mori, E., 2000. Impact of white matter changes on clinical manifestation of Alzheimer's disease: a quantitative study. Stroke 31, 2182-2188.

Ho, B.C., Andreasen, N.C., Nopoulos, P., Arndt, S., Magnotta, V., Flaum, M., 2003. Progressive structural brain abnormalities and their relationship to clinica outcome: a longitudinal magnetic resonance imaging study early in schizophrenia. Arch. Gen. Psychiatry 60, 585-594.

Huang, H., Zhang, J., Jiang, H., Wakana, S., Poetscher, L., Miller, M.I., van Zijl, C.M., Hillis, A.E., Wytik, R., Mori, S., 2005. DTI tractography based parcellation of white matter: application of the mid-sagittal morphology of corpus callosum. Neurolmage 26 (1), 295-305.

Huang, M., Theilmann, R.J., Robb, A., Angeles, A., Nichols, S., Drake, A., Dandrea, J., Levy M., Holland, M., Song, T., 2009. Integrated imaging approach with MEG and DTI to Detect mild traumatic brain injury in military and civilian patients. J. Neurotrauma 26 (8), 1213-1226.

Jones, D.K., Symms, M.R., Cercignani, M., Howard, R.J., 2005. The effect of filter size on VBM analyses of DT-MRI data. NeuroImage $26(2), 546-554$

Jones, D.K., Catani, M., Pierpaoli, C., Reeves, S.J., Shergill, S.S., O'Sullivan, M. Golesworthy, P., McGuire, P., Horsfield, M.A., Simmons, A., Williams, S.C., Howard, R.J., 2006. Age effects on diffusion tensor magnetic resonance imaging tractography measures of frontal cortex connections in schizophrenia. Hum. Brain Mapp. 27 (3) 230-238.

Kamada, K., Todo, T., Masutani, Y., Aoki, S., Ino, K., Morita, A., Saito, N., 2007. Visualization of the frontotemporal language fibers by tractography combined with functional magnetic resonance imaging and magnetoencephalography. J. Neurosurg. 106 (1), 90-98.

Kolmogorov, A.N., 1965. Three approaches to the quantitative definition of information. Inf. Trans. 1, 3-11.

Koukkou, M., Lehmann, D., Wackermann, J., Dvorak, I., Henggeler, B., 1993. Dimensional complexity of EEG brain mechanisms in untreated schizophrenia. Biol. Psychiatry $33(6), 397-407$.

Kraus, M.F., Susmatas, T. Caughlin, B.P., Walker, C.J., Sweeney, J.A., Little, D.M., 2007. White matter integrity and cognition in chronic traumatic brain injury: a diffusion tensor imaging study. Brain 130 (Pt 10), 2508-2519.

Le Bihan, D., van Zijl, P., 2002. From the diffusion coefficient to the diffusion tensor NMR Biomed. 15, 431-434.

Lempel, A., Ziv, J., 1976. On the complexity of finite sequences. IEEE Trans. Inf. Theory 22, 75-81.

Li, Y., Tong, S., Liu, D., Gai, Y., Wang, X., Wang, J., Qiu, Y., Zhu, Y., 2008. Abnormal EEG complexity in patients with schizophrenia and depression. Clin. Neurophysiol. 119 (6), 1232-1241.

Lopes da Silva, F., 1991. Neural mechanisms underlying brain waves: from neura membranes to networks. Electroencephalogr. Clin. Neurophysiol. 79 (2), 81-93.

Lutzenberger, W., Preissl, H., Pulvermuller, F., 1995. Fractal dimension of electroencephalographic time series and underlying brain processes. Biol. Cybern. 73 (5) $477-482$.

Markowithsch, H.J., 1995. Which brain regions ate critically involved in the retrieval of old episodic memory? Brain Res. Brain Res. Rev. 21 (2), 117-127.

McLaughlin, N.C., Paul, R.H., Grieve, S.M., Williams, L.M., Laidlaw, D., DiCarlo, M., Clark, C.R., Whelihan, W., Cohen, R.A., Whitford, T.]., Gordon, E., 2007. Diffusion tensor imaging of the corpus callosum: a cross-sectional study across the lifespan. Int. J. Dev. Neurosci. 25 (4), 215-221.

Meyer-Lindenberg, A., 1996. The evolution of complexity in human brain development: an EEG study. Electroencephalogr. Clin. Neurophysiol. 99 (5), 405-411. 
Mori, S., Wakana, S., Nagae-Poetscher, L.M., Van Zijl, P.C., 2005. MRI Atlas of Human White Matter. Elsevier, Amsterdam.

Nagarajan, R., 2002. Quantifying physiological data with Lempel-Ziv complexitycertain issues. IEEE Trans. Biomed. Eng. 49 (11), 1371-1373.

Oouchi, H., Yamada, K., Sakai, K., Kizu, O., Kubota, T., Ito, H., Nishimura, T., 2007. Diffusion anisotropy measurement of brain white matter is affected by voxel size: understimation occurs in areas with crossing fibers. AJNR Am. J. Neuroradiol. 28, 1102-1106.

Pereda, E., Quiroga, R.Q., Bhattacharya, J., 2005. Nonlinear multivariate analysis of neurophysiological signals. Prog. Neurobiol. 77 (1-2), 1-37.

Pfurtscheller, G., Lopes da Silva, F.H., 1999. Event-related EEG/MEG synchronization and desynchronization: basic principles. Clin. Neurophysiol. 110 (11), 1842-1857.

Radhakrishnan, N., Gangadhar, B.N., 1998. Estimating regularity in epileptic seizure timeseries data. A complexity-measure approach. IEEE Eng. Med. Biol. Mag. 17 (3), 89-94.

Raichle, M.E., Macleod, A.M., Snyder, A., Powers, W.J., Gusnard, D.A., Shulman, G.L 2001. A default mode of brain function. Proc. Natl. Acad. Sci. 98, 676-682.

Roberts, T.P., Khan, S.Y., Blaskey, L., Dell, J., Levy, S.E., Zarnow, D.M., Edgar, J.C., 2009. Developmental correlation of diffusion anisotropy with auditory-evoked response. Neuroreport 20 (18), 1586-1591.

Robinson, P.A., Rennie, C.J., Wright, J.J., Bahramali, H., Gordon, E., Rowe, D.L., 2001. Prediction of electroencephalographic spectra from neurophysiology. Phys. Rev. E Stat. Nonlin. Soft. Matter. Phys. 63 (2 Pt 1), 021903.

Salat, D.H., Tuch, D.S., Greve, D.N., van der Kouwe, A.J., Hevelone, N.D., Zaleta, A.K. Rosen, B.R., Fischl, B., Corkin, S., Rosas, H.D., Dale, A.M., 2005. Age-related alterations in white matter microstructure measured by diffusion tensor imaging. Neurobiol. Aging 26, 1215-1227.

Schmahmann, J., Pandya, D., 2006. Fiber Pathways of the Brain. Oxford University Press, Oxford.

Segalowitz, S.J., Santesso, D.L., Jetha, M.K., 2010. Electrophysiological changes during adolescence: a review. Brain Cogn. 72 (1), 86-100.

Shaw, P., Eckstrand, K., Sharp, W., Blumenthal, J., Lerch, J.P., Greenstein, D., Clasen, L., Evans, A., Giedd, J., Rapoport, J.L, 2007. Attention-deficit/hyperactivity disorder is characterized by a delay in cortical maturation. Proc Natl. Acad. Sci. 104 (49), 19649-19654.

Shimony, J.S., McKinstry, R.C., Akbudak, E., Aronovitz, J.A., Snyder, A.Z., Lori, N.F., Cull, T.S Conturo, T.E., 1999. Quantitative diffusion-tensor anisotropy brain MR imaging. normative human data and anatomic analysis. Radiology 212 (3), 770-784.

Sowvell, ER Thompson, PM, Holmes, CJ Batth, R, Jernigan, TI Toga, AW 1999. Localizing age-related changes in brain structure between childhood and adolescence using statistical parametric mapping. Neurolmage 9 (6 Pt 1), 587-597.
Sporns, O., Tononi, G., Edelman, G.M. 2000. Connectivity and complexity: the relationship between neuroanatomy and brain dynamics. Neural Netw. 13 (8-9), 909-922.

Stam, C.J., 2005. Nonlinear dynamical analysis of EEG and MEG: review of an emerging field. Clin. Neurophysiol. 116 (10), 2266-2301.

Stam, C.J., Jones, B.F., Nolte, G., Breakspear, M., Scheltens, P., 2007. Small-world networks and functional connectivity in Alzheimer's disease. Cereb. Cortex 17, 92-99.

Steen, R.G., Ogg, R.J., Reddick, W.E., Kingsley, P.B., 1997. Age-related changes in the pediatric brain: quantitative MR evidence of maturational changes during adolescence. AJNR Am. J. Neuroradiol. 18 (5), 819-828.

Stufflebeam, S.M., Witzel, T., Mikulski, S., Hamalainen, M.S., Temereanca, S., Barton, J.J., Tuch, D.S. Manoach, D.S., 2008. A non-invasive method to relate the timing of neural activity to white matter microstructural integrity. Neurolmage 42 (2), $710-716$

Tononi, G., Sporns, O., Edelman, G.M., 1994. A measure for brain complexity: relating functional segregation and integration in the nervous system. Proc. Natl. Acad. Sci. U. S. A. 91 (11), 5033-5037.

Tononi, G., Edelman, G.M., Sporns, O., 1998. Complexity and coherency: integrating information in the brain. Trends Cogn. Sci. 2 (12), 474-484

Wakana, S., Jiang, H., Nagae-Poetscher, L.M., van Zijl, P.C., Mori, S., 2004. Fiber tract-based atlas of human white matter anatomy. Radiology $230(1), 77-87$.

Whitford, T.J., Rennie, C.J., Grieve, S.M., Clark, C.R., Gordon, E., Williams, L.M., 2007. Brain maturation in adolescence: concurrent changes in neuroanatomy and neurophysiology. Hum. Brain Mapp. 28 (3), 228-237.

Widjaja, E., Zarei Mahmoodabadi, S., Otsubo, H., Snead, O.C., Holowka, S., Bells, S., Raybaud, C., 2009. Subcortical alterations in tissue microstructure adjacent to focal cortical dysplasia: detection at diffusion-tensor MR imaging by using magnetoencephalographic dipole cluster localization. Radiology 251 (1), 206-215.

Widjaja, E., Simao, G., Mahmoodabadi, S.Z., Ochi, A., Snead, O.C., Rutka, J., Otsubo, H., 2010. Diffusion tensor imaging identifies changes in normal-appearing white matter within the epileptogenic zone in tuberous sclerosis complex. Epilepsy Res. $89(2-3), 246-253$

Yan, R., Gao, R.X., 2004. Complexity as a measure for machine health evaluation. IEEE Trans. Instrum. Meas. 53 (4), 1327-1334.

Zarei, M., Johansen-Berg, H., Smith, S., Ciccarelli, O., Thompson, A.J., Matthews, P.M., 2006. Functional anatomy of interhemispheric cortical connections in the human brain. J. Anat. 209 (3), 311-320.

Zhang, X.S., Roy, R.J., Jensen, E.W., 2001. EEG complexity as a measure of depth of anesthesia for patients. IEEE Trans. Biomed. Eng. 48 (12), 1424-1433. 